\title{
Effects of band offset and nonparabolicity on the effective mass of two-dimensional electron gases in modulation- $\delta$-doped $\mathrm{Ga}_{0.47} \mathrm{In}_{0.53} \mathrm{As}$-based heterostructures
}

\author{
Y. T. Dai and Y. F. Chen \\ Department of Physics, National Taiwan University, Taipei, Taiwan 107, Republic of China \\ I. Lo \\ Department of Physics, Sun Yat-Sen University, Kaohsiung, Taiwan, Republic of China
}

(Received 11 March 1996; revised manuscript received 5 November 1996)

\begin{abstract}
We present an investigation of electronic and optical properties of two-dimensional electron gases confined in modulation- $\delta$-doped $\mathrm{Ga}_{0.47} \mathrm{In}_{0.53}$ As-based heterostructures by Shubnikov-de Haas measurement and optically detected cyclotron resonance (ODCR). Quantum oscillations clearly show the occupation of the first two subbands. The values of the effective masses obtained from ODCR measurements are considerably higher than the bulk data by $20-50 \%$. The theoretical prediction of the effect of nonparabolicity is insufficient to explain the experimental results. By choosing heterostructures with a very large difference in the conduction-band offset, we clearly show that this discrepancy can be attributed to the effect of wave-function penetration into the barrier material. [S0163-1829(97)02608-8]
\end{abstract}

The value of effective mass is a very important parameter in the determination of semiconductor properties, such as mobility, transport properties, electronic states, and optical transitions. $\mathrm{In}_{x} \mathrm{Ga}_{1-x}$ As-based quantum structures are of great interest for applications in high-speed electronic and optoelectronic devices. To date, the realization of high twodimensional carrier concentration in this material system has been reported. ${ }^{1-4}$ The effective mass used in the calculation of various transport properties of such structures is often approximated by the corresponding value for bulk material. ${ }^{1}$ However, this assumption becomes less valid with increasing Fermi energy. It is known, for instance, that the in-plane effective masses are expected to exceed the bulk values considerably due to the increasing effect of band nonparabolicity at higher Fermi energy. 5 ,6 The experimental verification of these effects is of great importance. Even though several reports have been devoted to the study of this problem, the effective mass including nonparabolic correction is always less than the experimental result. ${ }^{7-11}$ It has been pointed out that this discrepancy can be attributed to the effect of the wave-function penetration into the barrier; 9,11 however, there is still no clear evidence to demonstrate the penetration effect. In this paper we present direct measurements of the in-plane effective mass of the two-subband occupied electron gas in modulation- $\delta$-doped $\mathrm{Ga}_{1-x} \mathrm{In}_{x}$ As-based heterostructures with a very large difference of conduction-band offset by using far-infrared (FIR) optically detected cyclotron resonance (ODCR). We are able to directly resolve the contribution of band nonparabolicity and the wave-function penetration into the barrier to the enhanced effective masses.

The modulation- $\delta$-doped $\operatorname{In}_{0.53} \mathrm{Ga}_{0.47} \mathrm{As}$ heterostructures studied in this work were grown by molecular beam epitaxy on semi-insulating Fe-doped InP substrates. One consists of $350 \mathrm{~nm}$ of $\operatorname{In}_{0.52} \mathrm{Al}_{0.48} \mathrm{As}, 50 \mathrm{~nm}$ of $\operatorname{In}_{0.53} \mathrm{Ga}_{0.47} \mathrm{As}$ quantum well layer, $36 \mathrm{~nm}$ of $\operatorname{In}_{0.52} \mathrm{Al}_{0.48} \mathrm{As}$, capped by $5 \mathrm{~nm}$ of GaAs. The other is $80 \mathrm{~nm}$ of $\mathrm{AlAs}_{0.56} \mathrm{Sb}_{0.44}, 50 \mathrm{~nm}$ of $\mathrm{In}_{0.53} \mathrm{Ga}_{0.47} \mathrm{As}$, and $26 \mathrm{~nm}$ of $\mathrm{AlAs}_{0.56} \mathrm{Sb}_{0.44}$. In addition, a $220-\mathrm{nm} \mathrm{In} \mathrm{In}_{0.52} \mathrm{Al}_{0.48} \mathrm{As}$ buffer layer was added on the bottom and another $12.5-\mathrm{nm} \operatorname{In}_{0.52} \mathrm{Al}_{0.48} \mathrm{As}$ layer was placed on the top before the GaAs cap. The silicon $\delta$-doped layer is $6 \mathrm{~nm}$ away from the $\operatorname{In}_{0.53} \mathrm{Ga}_{0.47} \mathrm{As}$ layer with a doping concentration of about $(3-4) \times 10^{12} \mathrm{~cm}^{-2}$ for both samples. Therefore, the $\operatorname{In}_{x} \mathrm{Ga}_{1-x}$ As heterostructures have the same well parameters [e.g., $N_{d}=(3-4) \times 10^{12} \mathrm{~cm}^{-2}, s=6 \mathrm{~nm}$, and $L_{z}=50 \mathrm{~nm}$ ], but have very different conduction-band offsets. For the $\mathrm{In}_{0.52} \mathrm{Al}_{0.48} \mathrm{As} / \mathrm{In}_{0.53} \mathrm{Ga}_{0.47} \mathrm{As}$ heterostructure, the conductionband offset is $\Delta E_{c}=0.586 \mathrm{eV},{ }^{12}$ and for the $\mathrm{AlAs}_{0.56} \mathrm{Sb}_{0.44} / \mathrm{In}_{0.53} \mathrm{Ga}_{0.47} \mathrm{As}$ heretostructure, it has $\Delta E_{c}=1.75 \mathrm{eV} .{ }^{13}$ In these two heterostructures, because only one barrier of the quantum well is doped, the symmetric square well turns into a triangular well due to band bending at the interface. Thus most electrons transferred from the doped barrier are confined in the triangular well, and the undoped barrier hardly contributes to the confinement of the electrons.

The electronic properties of the studied heterostructures were characterized by Shubnikov-de Haas $(\mathrm{SdH})$ measurements. This measurement can be used to determine the individual electron concentrations of a multiple carrier system. Also, it can provide the evidence to show the occupation of two-dimensional electron gas (2DEG) in the first two subbands in our studied samples, which is important for our ODCR measurement. The $\mathrm{SdH}$ measurements of the InAlAs $/ \operatorname{In}_{x} \mathrm{Ga}_{1-x} \mathrm{As}$ and $\mathrm{AlAsSb} / \mathrm{In}_{x} \mathrm{Ga}_{1-x} \mathrm{As}$ heretostructures are shown in Figs. 1(a) and 1(b), respectively. Each spectrum clearly exhibits two oscillations corresponding to the occupation of the first and second subbands. From the fast Fourier transform spectrum, the electron concentrations obtained in the InAlAs/ $\operatorname{In}_{x} \mathrm{Ga}_{1-x}$ As heterostructure are 17.3 and $3.6 \times 10^{11} \mathrm{~cm}^{-2}$ for the first and second subband, respectively. For the AlAsSb/In $\mathrm{Ga}_{1-x}$ As heterostructure, the electron concentrations of the first and second subbands are 26.8 and $6.5 \times 10^{11} \mathrm{~cm}^{-2}$, respectively. Using the effective masses obtained from our ODCR measurements, the calculated Fermi energies of the $\mathrm{InAlAs} / \mathrm{In}_{x} \mathrm{Ga}_{1-x} \mathrm{As}$ heterostructure are $E_{F 0}=70 \mathrm{meV}$ and $E_{F 1}=18 \mathrm{meV}$ for the first and second 

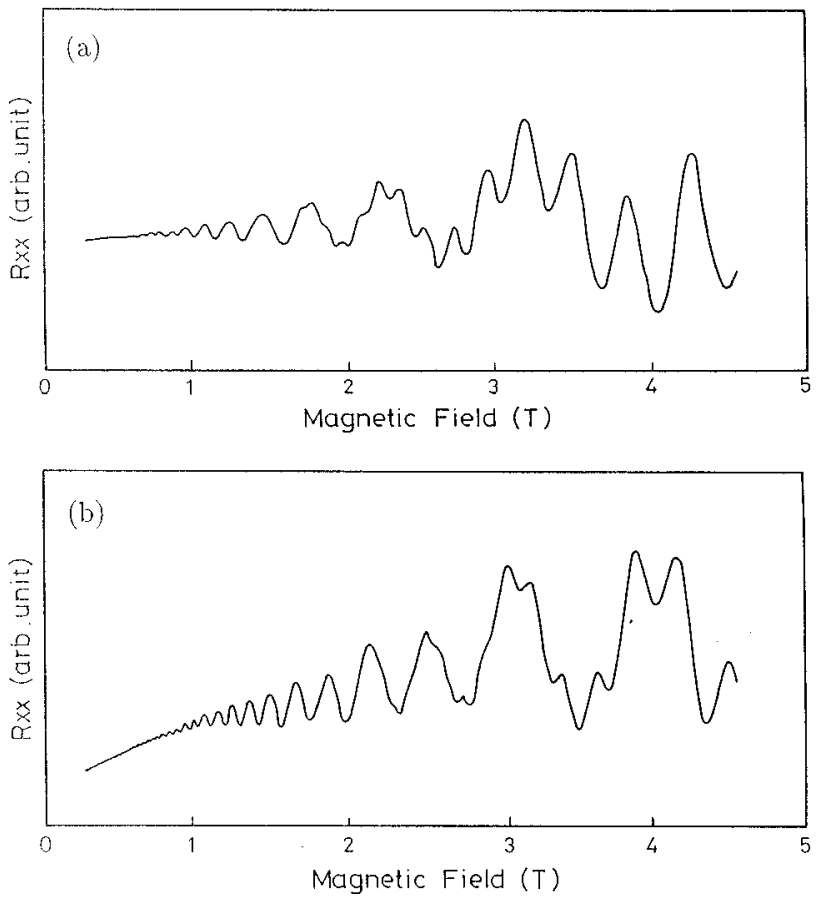

FIG. 1. The magnetoresistance $\left(R_{x x}\right)$ plotted against the magnetic field (a) for the $\mathrm{Al}_{0.48} \mathrm{In}_{0.52} \mathrm{As} / \mathrm{Ga}_{0.47} \mathrm{In}_{0.53} \mathrm{As}$ heterostructure at $4.2 \mathrm{~K}$, (b) for the $\mathrm{AlAs}_{0.56} \mathrm{Sb}_{0.44} / \mathrm{In}_{0.53} \mathrm{Ga}_{0.47} \mathrm{As}$ heterostructure at 5 $\mathrm{K}$.

subband, respectively, while $E_{F 0}=103 \mathrm{meV}$ and $E_{F 1}=31$ $\mathrm{meV}$ for the AlAsSb/In $\mathrm{Ga}_{1-x}$ As heterostructure. In the calculation of each Fermi energy, the assumption of a constant effective mass has been made. Because the band structures of the materials studied here are nonparabolic, one may expect that the calculated values are higher than the actual Fermi energies. However, according to the previous reports on $\mathrm{In}_{x} \mathrm{Ga}_{1-x}$ As heterostructures, ${ }^{14,15}$ the calculated results are consistent with the measured Fermi energies. We thus believe that the assumption of a constant effective mass is acceptable.

The effective mass for each subband was determined by ODCR measurements. In an ODCR measurement, cyclotron resonance (CR) is detected by observing changes in photoluminescence (PL) caused by cyclotron heating or cyclotroninduced impact ionization of bound carriers. ${ }^{16-18}$ Thus CR detection can employ the advantages of using highly sensitive optical detectors, and it can be studied in a thin epitaxial layer. In our measurements, the PL signals were performed using an Ar ion laser. The sample was placed in a 7-T superconducting magnet. The luminescence signal was dispersed by a $0.5-\mathrm{m}$ monochromator and detected by a liquidnitrogen-cooled Ge detector. For the ODCR experiment, the sample was simultaneously irradiated by a $\mathrm{CO}_{2}$-pumped FIR laser working at $118.8 \mu \mathrm{m}$. The FIR radiation was guided by mirrors to the sample and was modulated by a mechanical chopper. A detailed description of the apparatus has been given elsewhere. ${ }^{19}$

The ODCR spectra of the same InAlAs/ $/ n_{x} \mathrm{Ga}_{1-x}$ As heterostructure have been reported in our recent publication. ${ }^{20}$ Here, we will briefly summarize the result. It was found that the ODCR signal is structured by quantum oscillations,

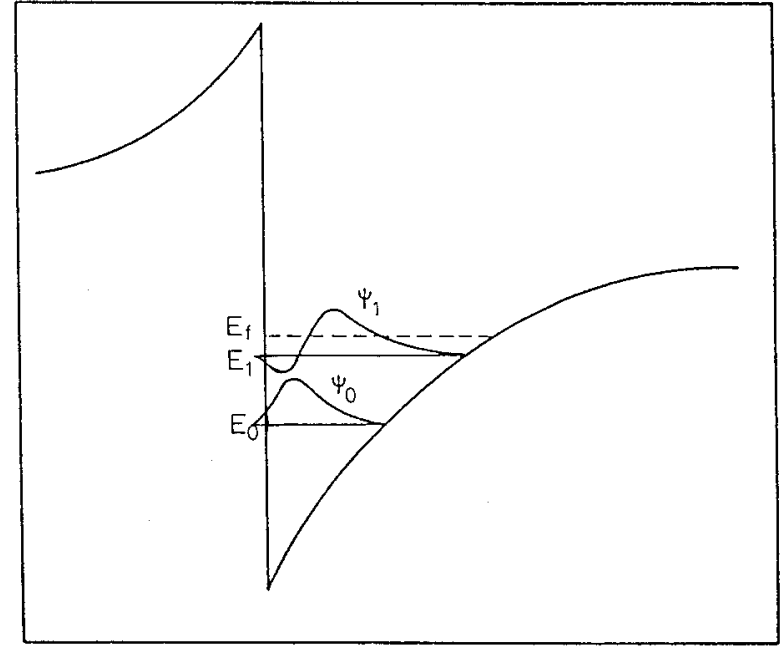

FIG. 2. The schematic diagram of the electronic structure and wave functions of a triangular well. $\psi_{0}$ and $\psi_{1}$ are the envelope wave functions of the first and second subbands, respectively.

which are induced by the crossing between the Landau levels of the 2DEG and the Fermi level. The spectrum contains two sets of oscillations, corresponding to an occupation of the first two subbands. The electron concentrations deduced from the period of $1 / B$ are 19 and $4.7 \times 10^{11} \mathrm{~cm}^{-2}$ for the first and second subbands, respectively, which are in good agreement with the SdH result. The ODCR spectrum can be fitted quite well by two classical Lorentzian functions, from which the CR fields can be determined. The effective masses obtained from the CR fields at $4.3 \mathrm{~K}$ are $0.060 m_{e} \pm 0.001 m_{e}$ and $0.049 m_{e} \pm 0.001 m_{e}$ for the first and second subband, respectively. This result is consistent with the measurement by conventional FIR Fourier transform CR experiment for a sample cut from the same wafer. ${ }^{21}$ To confirm that the carriers are two dimensional, a tilted angle experiment has been performed, and it shows that the CR line shifted with $B \cos \theta{ }^{21}$ The values of the effective masses obtained are considerably higher than the band-edge value of $m^{*}=0.041 m_{e}$ in the undoped bulk $\operatorname{In}_{0.53} \mathrm{Ga}_{0.47}$ As material measured under the same experimental condition. ${ }^{19}$

To interpret the enhancement of the effective masses, we first consider the effect of band nonoparabolicity. Quantitatively, the fact that the effective mass of the first subband is heavier than that of the second subband can be understood according to the concept of nonparabolicity. Figure 2 shows the schematic diagram of the heterojunction triangular potential profile and the wave functions of the first and second subbands. On the average, the electrons in the first subband are closer to the interface and in turn effectively at higher energy from the band edge, thus they will have a more enhanced mass than the electrons in the second subband. The quantitative evaluation of band nonparabolicity in a triangular well can be obtained according to Ando formulation, ${ }^{22}$

$$
\Delta m^{*} / m^{*}=\left[1+4\left(\langle K\rangle_{i}+E_{F i}\right) / E_{g}\right]^{1 / 2}-1,
$$

where $\langle K\rangle_{i}$ and $E_{F i}$ are the kinetic energy and the Fermi energy in the $i$ th subband, respectively, and $\langle K\rangle_{i}$ is given approximately as $E_{i} / 3$ for the triangular well. $E_{i}$ is the en- 
ergy of the $i$ th subband, and $E_{g}$ is the energy gap. If we take the values of the Fermi energies $E_{F 0}=70 \mathrm{meV}$ and $E_{F 1}=18$ $\mathrm{meV}$, from our $\mathrm{SdH}$ measurements, $E_{g}=0.81 \mathrm{eV},{ }^{23}$ and the minimum energy of the first subband $E_{0}$ is approximately equal to $110 \mathrm{meV}$ as calculated from the simple Hartree expression, ${ }^{7,24}$ the estimated effective masses are $0.051 m_{e}$ and $0.048 m_{e}$ for the first and second subband, respectively. If we take the carrier concentrations for the sample under illumination obtained from the ODCR measurement or from our previous publication, ${ }^{25}$ the estimated effective masses for the first and second subband are enhanced by about $0.0005 m_{e}$ compared with the result for the sample without illumination. Thus the effect of illumination can be neglected. The fact that the effective mass of the first subband is heavier than that of the second subband can be explained quite well, however, the experimental value of the first subband is significantly heavier than that of the theoretical prediction. If we adapt the concept of the penetration of the wave function into the barrier material, the significant deviation of the effective mass of the first subband from the nonparabolic correction can be understood according to the schematic diagram shown in Fig. 2. Because the electrons in the first subband are closer to the interface their wave function has a larger penetration into the barrier layer, and hence they will have a more enhanced mass than the electrons in the second subband. In order to confirm the important role of the wave-function penetration on the determination of the effective mass, we perform the same experiments on a similar structure, but the $\operatorname{In}_{0.52} \mathrm{Al}_{0.48} \mathrm{As}$ barrier layer is now replaced by an $\mathrm{AlAs}_{0.56} \mathrm{Sb}_{0.44}$ layer. This replacement will change the conduction-band offset from 0.586 to $1.75 \mathrm{eV}$. Since the wave-function penetration into the barrier material decreases with increasing barrier height, we shall expect that the larger barrier height will result in a smaller enhanced effective mass under the condition of the same carrier concentration.

The near-band-edge photoluminescence spectrum of the AlAsSb/ $\mathrm{In}_{x} \mathrm{Ga}_{1-x}$ As sample consists of a broad line located at $0.793 \mathrm{eV}$, which may be attributed to the unresolved contributions of free excitons, bound excitons, and/or donor-tofree-hole recombinations. ${ }^{26,27}$ The FIR-induced PL change monitored at the peak energy as a function of magnetic field is shown in Fig. 3. Again, a strong ODCR signal is observed, but the quantum oscillations are much less pronounced than that in the InAlAs/ $/ \operatorname{In}_{x} \mathrm{Ga}_{1-x} \mathrm{As}$ heterostructure. It probably indicates that the InAlAs/ $/ n_{x} \mathrm{Ga}_{1-x}$ As heterostructure has a better sample quality. Figure 3 also shows the ODCR spectra at different temperatures, which clearly show that the relative CR amplitude of the second subband to that of the first subband increases with increasing temperature. Each CR spectrum can also be fitted quite well by two Lorentzian functions. From the CR fields at $5 \mathrm{~K}$, the obtained effective masses of the first subband and second subband are $0.057 m_{e} \pm 0.001 m_{e}$ and $0.050 m_{e} \pm 0.002 m_{e}$, respectively. Using Eq. (1), the theoretical values of the effective masses including the effect of nonparabolicity for the first and second subbands are $0.054 m_{e}$ and $0.050 m_{e}$, respectively. These values are in good agreement with the experimental result. Similar to the InAlAs $/ \mathrm{In}_{x} \mathrm{Ga}_{1-x}$ As sample, we can also estimate the effect of illumination. In a previous report, ${ }^{25}$ illuminating the AlAsSb/In ${ }_{x} \mathrm{Ga}_{1-x}$ As sample at $4.2 \mathrm{~K}$, the carrier concentrations increase from 26.8 to $30.1 \times 10^{11} \mathrm{~cm}^{-2}$

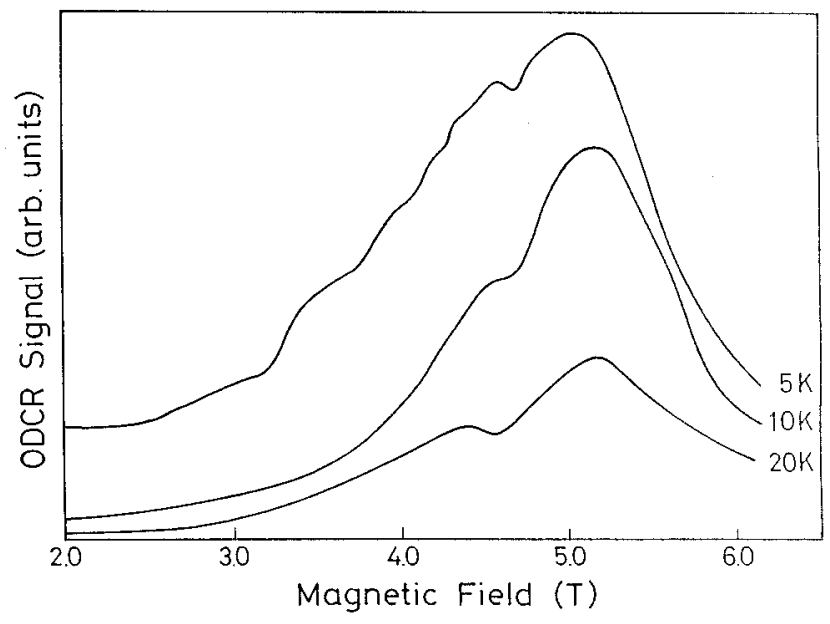

FIG. 3. The ODCR spectra of the $\mathrm{AlAs}_{0.56} \mathrm{Sb}_{0.44} / \mathrm{In}_{0.53} \mathrm{Ga}_{0.47} \mathrm{As}$ heterostructure at different temperatures.

and from 6.5 to $7.3 \times 10^{11} \mathrm{~cm}^{-2}$ for the first and second subband, respectively. Using the increased carrier concentrations by illumination, the estimated effective masses can only be enhanced by $0.0008 m_{e}$ and $0.0004 m_{e}$ for the first and second subband, respectively, when compared with the result for the sample without illumination. Thus, as in the InAlAs $/ \mathrm{In}_{x} \mathrm{Ga}_{1-x} \mathrm{As}$ sample, the effect of illumination is negligible. It is interesting to note that the carrier concentration of the AlAsSb/In $\mathrm{Ga}_{1-x} \mathrm{As}$ sample is higher than that of the InAlAs $/ \operatorname{In}_{x} \mathrm{Ga}_{1-x} \mathrm{As}$ sample, thus according to the effects of nonparabolicity or band filling, the former sample should have a more enhanced effective mass of the Fermi electrons, which is in contrast with the experimental result for the first subband. However, this reversed behavior is consistent with the prediction of the effect of the wave-function penetration into the barrier material. Because the main difference between the InAlAs $/ \mathrm{In}_{x} \mathrm{Ga}_{1-x}$ As and AlAsSb/In $\mathrm{Ga}_{1-x}$ As heterostructures is that the latter sample has a much larger conduction-band offset, it has a much less enhanced effective mass due to the effect of the wave-function penetration into the barrier. We therefore can conclude that the enhanced effective masses of the 2DEG in the AlAsSb/ $\operatorname{In}_{x} \mathrm{Ga}_{1-x} \mathrm{As}$ heterostructure studied here are mainly induced by the effect of band nonparabolicity because of its large conduction-band offset. The wave-function penetration into the barrier material does play a very important role in the determination of the effective mass for the InAlAs/ $\mathrm{In}_{x} \mathrm{Ga}_{1-x}$ As heterostructure due to its small conduction-band offset.

In summary, we have attempted to resolve the origins of the enhancement of the effective mass in two-subband occupied 2DEG systems. Using the ODCR technique, quite different effective masses associated with the first and second subbands are obtained. It is found that even though the theoretical calculation including band nonparabolicity can account for the fact of the effective mass of the first subband being heavier than that of the second subband, it fails to predict the large value of the first subband. This discrepancy is attributed to the effect of the wave-function penetration into the barrier material. We have clearly demonstrated this effect by performing the same experiments on heterostructures having a very large difference of conduction-band off- 
set. It is concluded that except for the effect of band nonparabolicity, the wave-function penetration into the barrier material does play a very important role in the determination of the enhanced effective mass.
This work was partly supported by National Science Council of the Republic of China. The authors are grateful to Dr. W. C. Mitchel, Dr. P. W. Yu, Dr. A. Fathimulla, and Dr. H. Hier for providing the samples studied.
${ }^{1}$ U. Wiesner, J. Pillath, A. Kohl, A. M. Küsters, S. Brittner, and K. Heime, Appl. Phys. Lett. 64, 2520 (1994).

${ }^{2}$ C. E. Stutz, B. Jogai, D. C. Look, J. M. Ballingall, and T. J. Roger, Appl. Phys. Lett. 64, 2703 (1994).

${ }^{3}$ I. Lo, W. C. Mitchel, M. Ahoujja, J. P. Cheng, A. Fathimulla, and H. Hier, Appl. Phys. Lett. 66, 754 (1995).

${ }^{4}$ Y. F. Chen, J. L. Shen, Y. D. Dai, G. J. Jan, and H. H. Lin, Appl. Phys. Lett. 66, 2543 (1995).

${ }^{5}$ G. Bastard, J. A. Brum, and R. Ferreira, in Solid State Physics: Advances in Research and Applications, edited by H. Ehrenreich and D. Turnbull (Academic, New York, 1991), Vol. 44, p. 229.

${ }^{6}$ U. Ekenberg, Phys. Rev. B 40, 7714 (1989).

${ }^{7}$ M. A. Brummell, R. J. Nicholas, L. C. Brunel, S. Huant, M. Baj, J. C. Portal, M. Razeghi, M. A. Di Forte-Poisson, K. Y. Cheng, and A. Y. Cho, Surf. Sci. 142, 380 (1984).

${ }^{8}$ S. Yamada, T. Fukui, K. Tsubaki, and A. Sugimura, Phys. Rev. B 32, 8078 (1985).

${ }^{9}$ C. Wetzel, Al. L. Efros, A. Moll, B. K. Meyer, P. Omling, and P. Sobkowicz, Phys. Rev. B 45, 14052 (1992).

${ }^{10}$ Y. F. Chen, J. L. Shen, Y. D. Dai, and F. F. Fang, Phys. Rev. B 52, 4692 (1995).

${ }^{11}$ B. R. Nag and S. Mukhopadhyay, Appl. Phys. Lett. 62, 2416 (1993).

${ }^{12}$ R. Sauer, T. D. Harris, and W. T. Tsang, Phys. Rev. 34, 9023 (1986)

${ }^{13}$ Y. Nakata, Y. Sugiyama, T. Inata, O. Ueda, S. Sasa, S. Muto, and T. Fujji, in Epitaxial Heterostructures, edited by D. W. Shaw et al., MRS Symposia Proceedings No. 198 (Materials Research Society, Pittsburgh, 1990), p. 289.
${ }^{14}$ L. V. Butov, V. D. Kulakovskii, T. G. Andersson, and Z. G. Chen, Phys. Rev. B 42, 9472 (1990).

${ }^{15}$ Y. H. Zhang, R. Cingolani, and K. Ploog, Phys. Rev. B 44, 5958 (1991).

${ }^{16}$ P. G. Baranov, Yu. P. Veshchunov, R. A. Zhitnikov, N. G. Romanov, and Yu. G. Shreater, Pis'ma Zh. Eksp. Teor. Fiz. 26, 369 (1977) [JETP Lett. 26, 249 (1977)].

${ }^{17}$ R. Romestain and C. Weisbuch, Phys. Rev. Lett. 45, 2067 (1980).

${ }^{18}$ M. G. Wright, N. Ahmed, A. Koohian, K. Mitchell, G. R. Johnson, B. C. Cavenett, C. R. Pidgeon, C. R. Stanley, and A. H. Kean, Semicond. Sci. Technol. 5, 438 (1990).

${ }^{19}$ J. L. Shen, Y. D. Dai, Y. F. Chen, S. Z. Chang, and S. C. Lee, Phys. Rev. B 51, 17648 (1995).

${ }^{20}$ Y. F. Chen, Y. T. Dai, and I. Lo, Appl. Phys. Lett. 68, 1117 (1996).

${ }^{21}$ J. P. Chang, I. Lo, W. C. Mitchel, and A. Fathimulla, Bull. Am. Phys. Soc. 40, 19 (1995).

${ }^{22}$ T. Ando, J. Phys. Soc. Jpn. 51, 3893 (1982).

${ }^{23}$ G. Livescu, D. A. B. Miller, D. S. Chemla, M. Ramaswamy, J. Y. Chang, N. Sauer, A. C. Gossard, and J. H. English, IEEE. J. Quantum Electron. 24, 1677 (1988).

${ }^{24}$ T. Ando, A. B. Fowler, and F. Stern, Rev. Mod. Phys. 54, 437 (1982).

${ }^{25}$ I. Lo, W. C. Mitchel, P. W. Yu, A. Fathimulla, and H. Hier, J. Appl. Phys. 79, 9196 (1996).

${ }^{26}$ V. Swaminathan, R. A. Stall, A. T. Macrander, and R. J. Wunder, J. Vac. Sci. Technol. B 3, 1631 (1985).

${ }^{27}$ A. P. Roth, M. A. Sacilotti, R. A. Masut, D. Morris, J. Young, C. Lacelle, E. Fortin, and J. L. Brebner, Can. J. Phys. 67, 330 (1989). 